sur les mammifères est essentiel si l'on veut que les résultats aient une valeur, car, comme l'ont montré les travaux d'ORLA-JENSEN, les composés de lactose et d'acides-aminés qui se forment au cours de la stérilisation auraient une action stimulante sur un grand nombre de bactéries lactiques (mais défavorable sur d'autres).

Nous sommes donc en présence d'une question complexe où il est cependant permis de dire que le brunissement du lait s'accompagne d'un blocage-d'acides-aminés préjudiciable à la croissance du jeune mammifère et, qu'en conséquence, toute technique de stérilisation conduisant au brunissement du lait doit être considérée comme défavorable à la valeur nutritive de cet aliment.

Les données du problème de la stérilisation du lait sont donc bien différentes de celles de la stérilisation de la plupart des autres conserves alimentaires. A la condition impérative commune d'obtenir un produit aussi exempt de germes que possible, se superpose ici la nécessité absolue de réaliser cette stérilisation dans des temps aussi brefs que possible - dono à température aussi élevée que possible - pour éviter ou réduire au maximum des altérations de la couleur, de la saveur et des propriétés nutritives qui nuisent à la valeur commerciale et alimentaire du produit.

(A suivre.)

\title{
LES PROTIDES DU LAIT (1)
}

$$
\text { par }
$$

\section{GÉrard BISERTE, ANDRÉ BRETON et GUY FONTAINE}

Le lait, aliment exclusif du nouveau-né et du jeune nourrisson, possède un système protéique complexe, dont les constituants appartiennent à deux types principaux : la caséine et les protéines du lactosérum, globulines et albumines.

La répartition de ces groupes de protéines est très différente suivant l'origine du lait: dans le lait de vache, les quatre-cinquièmes sont constitués par de la caséine et le cinquième restant est présent dans le lactosérum. Dans le lait de femme, au contraire, le rapport caséine protéine du lactosérum est sensiblement de 1 . Ceci est très important, compte tenu de la valeur nutritive de la fraction albumine.

Cette "hétérogénéité de base " révélée par les méthodes chimiques, s'est encore accrue avec l'utilisation des techniques modernes

(1) Travail du Laboratoire de Biochimie ds la Faculté de Médecine et de Pharmacie de Lille (Professeur P. Boulanger). La Semaine des Hopitaux, 1955, 31, n०73-7, 393. 
de fractionnement des mélanges protéiques (relargages aux sels neutres, ultra-centrifugation, électrophorèse différentielle). Si bien que nous pouvons, dès maintenant, envisager l'existence de trois types au moins de caséine, de plusieurs "fractions globuliniques" et de trois composés albuminiques : $\beta$-lactoglobuline, $\alpha$-lactalbumine et sérumalbumine.

Nous étudierons d'abord de façon analytique chacun de ces composés, puis nous envisagerons, dans une étape synthétique, le comportement de ces différentes fractions dans le «lait total» et les associations qu'elles contractent entre elles.

\section{I. - LA CASÉtine}

C'est un phosphoprotéide, le plus caractéristique, le plus abondant et l'un des mieux connus des constituants protéiques du lait.

\section{A. Les méthodes de préparation}

Elles sont nombreuses et diffèrent suivant que l'on s'adresse au lait de vache ou au lait de femme.

En ce qui concerne le lait de vache, quelle que soit la méthode, le principe est toujours le même : précipitation sous forme de caillots volumineux, par abaissement du $p \mathrm{H}$ au voisinage du point isoélectrique de la caséine [4] [6].

La préparation de la caséine du lait de femme est beaucoup plus délicate : elle nécessite le chauffage à $40^{\circ} \mathrm{C}$. du lait acidifié et sa dilution, à plusieurs reprises, avec de l'eau. Nous avons, au cours de nos études électrophorétiques du lait de femme, précipité la caséine par le suc gastrique de nourrisson en utilisant l'activité enzymatique de la rennine ou lab-ferment (Mellander [1]).

\section{B. La composition élémentaire de la caséine}

Elle a été étudiée par de nombreux auteurs : elle diffère suivant l'origine du lait. La caséine humaine est moins riche en azote total $(14,9$ à $15,1 \%)$ que la caséine de vache $(15,6 \%)$. Elle contient moitié moins de phosphore $(0,45 \%)$ que la caséine de vache $(0,81 \%)$.

\section{Hétérogénéité de la caséine}

L'hétérogénéité de la caséine a été constatée d'abord par des méthodes chimiques. Puis, l'ultracentrifugation, tout en apportant des résultats assez disparates, a renforcé cette notion d'hétérogénéité, L'électrophorèse enfin, a confirmé la complexité de la caséine et a permis l'individualisation de trois fractions différentes, appelées $\alpha, \beta$ et $\gamma$-caséines dans l'ordre de leur mobilité électrophorétique décroissante.

Par des méthodes chimiques nombreuses (précipitation au 
point iso-électrique, précipitation fractionnée dans l'alcool à $50 \%$ en présence de sels, précipitation fractionnée par l'urée) ces trois fractions de caséine de vache ont été isolées dans les proportions suivantes :

$\alpha$-caséine : $75 \%$ de la caséine totale,

$\beta$-caséine : $22 \%$ de la caséine totale,

$\gamma$-caséine : $3 \%$ de la caséine totale.

Il n'a pas été possible de fractionner la caséine de femme par ces méthodes : seule l' $\alpha$-caséine humaine a pu être séparée par électrophorèse différentielle du type Tiselius.

\section{Propriétés générales des caséines}

\section{$1^{\circ}$ Propriétés physico-chimiques générales.}

Les données concernant le poids moléculaire des caséines sont encore assez confuses : les résultats varient, en effet, suivant les méthodes de préparation des $\alpha$-et $\beta$-caséines.

En ce qui concerne leurs propriétés chimiques générales, il est intéressant de constater que les taux de phosphore et de soufre sont très différents pour les trois fractions caséiniques de vache : la $\gamma$-caséine est la plus riche en soufre $(1,03 \%)$, l' $\alpha$-caséine la moins riche $(0,72 \%)$, tandis que la $\beta$-caséine a un taux moyen de $0,86 \%$; le taux de phosphore varie en sens inverse du taux de soufre : c'est $l^{\prime} \alpha$-caséine qui en possède le plus $(1 \%)$ et la $\gamma$-caséine le moins $(0,10 \%)$, avec des chiffres intermédiaires pour la $\beta$-caséine $(0,55$ à $0,60 \%$ ). Il y a, par ailleurs, un rapport direct entre la richesse en phosphore des fractions caséiniques et leur mobilité électrophorétique : les composés les plus riches en phosphore sont les plus rapides.

$2^{\circ}$ Composition en amino-acides des caséines.

La composition complète en amino-acides de l' $\alpha$-, de la $\beta$ - et de la $\gamma$-caséine du lait de vache a été établie par Gordon, SEmMETT et coll. [2] au moyen de techniques microbiologiques. Les valeurs sont rassemblées dans le tableau I.

La composition en acides aminés des caséines de vache révèle leur richesse en acide glutamique (22 gr. \% en moyenne pour les $\alpha$-, $\beta$ - et $\gamma$-caséines), en proline (16 gr. $\%$ en moyenne pour les $\beta$ - et $\gamma$-caséines), en leucine (12 gr. \% pour les $\beta$ - et $\gamma$-caséines) et la faible teneur ( $\alpha$-caséine), voire l'absence $(\beta$ - et $\gamma$-caséines) de cystine.

En ce qui concerne la caséine humaine, les données sont moins nombreuses et plus difficiles à interpréter parce que fournies par des expérimentateurs et des méthodes différents. Toutefois, il faut 
également signaler la richesse en acide glutamique (20,9\%). Les teneurs en tyrosine $(6,1 \mathrm{gr} . \%)$, en tryptophane $(1,05 \mathrm{gr} . \%)$, en méthionine $2 \mathrm{gr} .7 \%$ ), en cystine ( $0 \mathrm{gr}$. $6 \%$ ), en phénylalanine ( $5 \mathrm{gr} .8 \%$ ), en proline ( $8 \mathrm{gr} .9 \%$ ), en leucine $(12 \mathrm{gr} .2 \%$ )... et isoleucine $(6 \mathrm{gr} .3 \%)$.

Il est intéressant de signaler que les prolamines alcoolosolubles (hordéine de l'orge, zéine du maïs, gliadine du blé), qui sont les principales protéines de réserve du règne végétal et qui sont toutes dégradées et utilisées au cours de la germination de la graine, sont caractérisées également par leur richesse en acide glutamique, leucine et proline (l'hordéine contient $26 \mathrm{gr}$. $76 \%$ d'acide glutamique).

L'hydrolyse enzymatique de la caséine, au cours des phénomènes chimiques de la digestion, libérera done une quantité très importante d'acide glutamique, "acide aminé-clef», du métabolisme intermédiaire.

D'autre part, les caséines de vache sont pauvres en cystine. La majorité du soufre provient de la méthionine, acide aminé qui joue un rôle essentiel dans les trans-méthylations.

$3^{\circ}$ Données sur la structure chimique de la caséine.

a) Détermination des groupes $\alpha$-aminés libres. La détermination des groupes aminés terminaux libres de l' $\alpha$ - et de la $\beta$-caséine a été réalisée par Mellon, Korn et Hoover [3], au moyen de la technique des dinitrophényl-aminoacides de SANGER. Ces groupes aminés terminaux de l' $\alpha$ - et de la $\beta$-caséine appartiennent à l'arginine et à la lysine. Les proportions de ces résidus d'amino-acides sont différentes dans l' $\alpha$ - ( 7 groupes arginine pour 1 groupe lysine) et la $\beta$-caséine ( 2 groupes arginine pour 1 groupe lysine). En conclusion, les molécules de caséine sont done formées par l'association de plusieurs types de chaînes polypeptidiques;

b) Structure primaire de la caséine. Le groupement prosthétique de la caséine est l'acide phosphorique lié à certains acides aminés (sérine et, à un degré moindre, thréonine), sous forme d'esters phosphoriques de sérine (phosphosérine) ou de thréonine (phosphothréonine). Levene et HuLl [4] ont pu identifier, d'autre part, un enchaînement dipeptidique du type acide phosphosérylglutamique.

Après l'hydrolyse enzymatique de la caséine par les enzymes des sucs digestifs (pepsine et trypsine) on peut isoler de l'hydrolysat, des molécules peptidiques complexes que l'on appelle phosphopeptones, riches en phosphore $(4,5 \%)$ et contenant un taux élevé d'acide glutamique et de sérine. Elles représentent $50 \%$ du phosphore total de la caséine de vache et $70 \%$ du phosphore de la 
caséine humaine. Les sels de calcium de ces phosphopeptones sont solubles dans l'eau et ont une action prépondérante dans la régulation du métabolisme phosphocalcique du nourrisson. Leur assimilation, d'après MELlander et Isaksson [5], favoriserait la calcification des os d'enfants rachitiques.

Les phosphopeptones sont déphosphorylées dans le tube digestif sous l'action d'une phosphatase de la muqueuse intestinale. Cette déphosphorylation est particulièrement efficace sur les phosphopeptones de la caséine de vache. La résistance relative qu'offrent les phosphopeptones de la caséine humaine, à cette déphosphorylation expliquerait partiellement la supériorité du lait de femme dans l'alimentation de l'enfant.

In vitro, la déphosphorylation directe des caséines, sous l'action des phosphatases d'origine intestinale ou prostatique, a été étudiée par Perlmann [6]. La déphosphorylation de l' $\alpha$-caséine par une phosphatase prostatique atteint $40 \%$ du phosphore total. L'électrophorèse différentielle permet de suivre les étapes de cette réaction et de noter l'apparition de composés nouveaux $\alpha_{1}, \alpha_{2}, \alpha_{3}, \alpha_{4}, \alpha_{5}$ qui correspondent à des caséines plus ou moins déphosphorylées. La $\beta$-caséine, au contraire, résiste à cette déphosphorylation. Elle semble, de plus, protéger l' $l^{\prime}$-caséine contre l'action enzymatique des phosphatases : la caséine brute n'est done pas déphosphorylée.

Poursuivant ses travaux sur la structure des caséines, PerLMANN [7] a soumis l' $\alpha$-caséine de vache à l'action combinée de trois enzymes spécifiques : une phosphodiestérase à $p H$ H,2, une pyrophosphatase à $p \mathrm{H} 7,2$ et une phosphatase intestinale et prostatique à $p$ H 6 : dans ces conditions, tout le phosphore de la protéine est libéré. De plus, la preuve est faite que l' $\alpha$-caséine possède :

$-40 \%$ de son phosphore sous forme de monoester ;

- $20 \%$ sous forme de pyrophosphates;

$-40 \%$ sous forme de diesters phosphoriques.

La présence d'une liaison pyrophosphate dans l' $\alpha$-caséine permet d'ailleurs de calculer le poids moléculaire minimum de la caséine. Si le cinquième du phosphore est présent sous forme de pyrophosphate, liaison où interviennent 2 atomes de phosphore, le nombre total par molécule doit être 10 ou un multiple de 10. Le poids atomique du phosphore étant de 31 , le poids moléculaire minimum doit être voisin de 31.000 .

$4^{\circ}$ Caséine et rénnine.

La précipitation de la caséine du lait sous forme de caillots par la présure, est le résultat d'une réaction enzymatique encore mal connue. La rennine est responsable de cette coagulation : c'est le 
principe actif de la présure. Elle est sécrétée sous une formeinactive par les glandes du quatrième estomac du veau, mais elle est activée par l'acide chlorhydrique du suc gastrique. La rennine possède des propriétés protéolytiques voisines de celles de la pepsine, mais son $p \mathrm{H}$ optimum d'action est plus élevé : 3,7 au lieu de 1,8 pour la pepsine. Au $p \mathrm{H}$ 6,6 et en 30 minutes, elle dédouble l' $\alpha$-caséine en deux composants électrophorétiques $\alpha_{1}$ et $\alpha_{2}$ (NITSCHMANN et Lehmann [8]). Toutefois, le temps nécessaire à la réalisation de cette hydrolyse est supérieur au temps de coagulation du lait. Il est vraisemblable que celle-ci se déroule en deux stades : enzymatique et non-enzymatique.

\section{II. - LES PROTÉINES DU LACTOSÉRUM}

Après élimination de la caséine à son point isoélectrique, le liquide surnageant, appelé lactosérum ou petit-lait, contient plusieurs groupes de protéines.

Les méthodes physiques (ultracentrifugation) permettent d'isoler trois composés majeurs :

- fraction $\alpha: \alpha$-lactalbumine de KEKWICK ;

- fraction $\beta$ : $\beta$-lactoglobuline de PaLmer ;

- fraction $\gamma$ : globulines immunes.

Les données récentes de l'électrophorèse confirment ces conclusions et permettent d'isoler en plus la sérumalbumine et plusieurs composés non identifiés (composé III de MacMerkin [9]).

Il est classique de séparer les protéines du lactosérum en deux groupes, suivant qu'elles sont précipitées ou non par le sulfate d'ammonium : le groupe des "globulines " et celui des " albumines ».

\section{Les globulines immunes}

Elles prédominent dans le colostrum, mais existent également dans le petit-lait parfait où elles représentent $10 \%$ des protéines. Obtenues par précipitation fractionnée au sulfate d'ammonium (SмIтн [10]) ou par précipitation à l'alcool (méthode de BAIN et Deutsch [11]), ces globulines immunes se scindent par dialyse contre l'eau distillée en euglobulines et pseudo-globulines, suivant qu'elles sont insolubles ou solubles dans l'eau pure.

Leurs poids moléculaire moyen est de 180.000. Elles sont riches en thréonine, en proline, contiennent des glucides et présentent une nette analogie de composition avec les $\gamma$-globulines sériques (tableau I).

\section{Propriétés immuno-chimiques.}

Du point de vue immunologique, les études de Wells et Osborne [12], de Crowther et de Raistrick [13] montrent que, 
TABLEAU I GOMPOOSITION EN AGIDES AMINÉS DE PROTÉINES DU LAIT DE VACHES EN GRAMMES POUR 100 G. DE PROTÉINES

\begin{tabular}{|c|c|c|c|c|c|c|}
\hline & $\alpha$-caséine & $\beta$-caséine & $\gamma$-caséine & $\begin{array}{c}\text { Pseudo-globuline } \\
\text { colostrale }\end{array}$ & $\begin{array}{l}\text { Globuline } \\
\text { immune }\end{array}$ & \multirow{2}{*}{$\begin{array}{c}\text { B-lactoglobuline } \\
\text { d'après Moore } \\
\text { et Stein [36] }\end{array}$} \\
\hline . & \multicolumn{3}{|c|}{ d'après Gordon et coll. [2] } & d'après Smith $[10$ & et Hansen 17] & \\
\hline Azote total . . . . . . & 15,53 & 15,33 & 15,40 & 15,9 & 0 & 15,6 \\
\hline Phosphore total ....... & 0,99 & 0,61 & 0,11 & 0 & 0 & 0 \\
\hline Acide aspartique . . . . . & 8,4 & 4,9 & 4 & 9,4 & - & 11,52 \\
\hline Acide glutamique...... & 22,5 & 23,2 & 22,9 & 12,3 & - & 19,08 \\
\hline Glycocolle $\ldots \ldots \ldots \ldots$ & 2,26 & 1,56 & 1,5 & - & - & 1,39 \\
\hline Alanine $\ldots \ldots \ldots \ldots$ & 3,81 & 1,99 & 2,3 & - & - & 7,09 \\
\hline Valine . . . . . . . . . & 6,3 & 10,2 & 10,5 & 8,7 & 9,6 & 5,62 \\
\hline Isoleucine $\ldots \ldots \ldots \ldots$ & 6,4 & 5,5 & 4,4 & 4,2 & 3,03 & 5,86 \\
\hline Leucine $\ldots \ldots \ldots \ldots$ & $7, y$ & 11,6 & 12 & 8,5 & 9,6 & 15,5 \\
\hline Sérine $\ldots \ldots \ldots \ldots \ldots$ & 6,3 & 6,8 & 5,5 & - & - & 3,96 \\
\hline Thréonine $\ldots \ldots \ldots \ldots$ & 4,9 & 5,1 & 4,4 & 9 & 10,5 & 4,92 \\
\hline Tyrosine ............ & 8,1 & 3,2 & 3,7 & 6,7 & - & 3,64 \\
\hline Phénylalanine ....... & 4,6 & 5,8 & 5,8 & 3,9 & 3,88 & 3,78 \\
\hline Proline ............ & 8,4 & 16 & 17 & 10 & - & 5,14 \\
\hline Histidine $\ldots \ldots \ldots \ldots$ & 2,9 & 3,1 & 3,7 & 2,3 & 2,05 & 1,63 \\
\hline Lysine $\ldots \ldots \ldots \ldots$ & 8,9 & 6,5 & 6,2 & 6,1 & 6,8 & 12,58 \\
\hline Arginine $\ldots \ldots \ldots \ldots$ & 4,3 & 3,4 & 1,9 & 5,6 & 4,05 & 2,91 \\
\hline Méthionine .......... & 2,5 & 3,4 & 4,1 & 1,3 & 0,90 & 3,22 (Smith) \\
\hline Cystine-2 $\ldots \ldots \ldots \ldots$ & 0,43 & $0,0-0,1$ & 0,0 & - & 3,15 & 3,4 \\
\hline Tryptophane ......... & 1,6 & 0,05 & 1,2 & 3,2 & 2,7 & 1,94 (Brand) \\
\hline
\end{tabular}


seule parmi les protéines du lait, la globuline colostrale supporte les propriétés immunes de l'animal. Des cobayes de 300 grammes préparés par injection intra-péritonéale de globuline immune de colostrum, subissent 30 jours plus tard, une injection intraveineuse déclenchante de globuline colostrale et sérique. Dans les deux cas, on observe des réactions sévères (convulsions) pour une dose modérée $(0 \mathrm{mgr} .2$ à $0 \mathrm{mgr}, 4)$. Parfois, la mort survient pour une dose supérieure $(0 \mathrm{mgr}$. 5 à $0 \mathrm{mgr}$. 6). Ni la lactalbumine, ni la caséine n'entraînent un tel choe anaphylactique : seule, la globuline colostrale peut le faire. Il est, par ailleurs, impossible d'établir une différence notable entre les globulines sérique et colostrale, dans la qualité et.l'intensité de l'injection déclenchante.

Ainsi, dans le colostrum, e'est la fraction globulinique qui supporte l'immunité et qui peut la transmettre au nouveau-né. En effet, les nouveau-nés de nombreuses espèces animales ne possèdent pas d'anticorps dans le sang eirculant, à cause de l'impossibilité presque totale du passage des $\gamma$-globulines-anticorps de la mère à travers le placenta. Cependant, le nouveau-né de ces mêmes espèces animales peut acquérir un taux important d'anticorps, grâce à l'ingestion du colostrum maternel. L'expérience suivante de YOUNG et UNDERDAHL [14] est assez significative à eet égard. Trois truies sont immunisées juste avant la fécondation par inoculation intranasale du virus grippal du porc. A la naissance, les sérums mélangés de 28 nouveau-nés ne contiennent pas d'anticorps. $\mathrm{Au}$ contraire, 30 heures après le début de l'alimentation, un taux élevé d'anticorps peut être décelé dans les sérums des nouveau-nés. Ce titre diminue d'ailleurs progressivement dans les jours qui suivent. Orcutr et Howe [15] ont prouvé également le passage d'agglutinines naturelles de Brucella abortus d'une vache à son veau, par l'intermédiaire des globulines du colostrum : quelques heures après l'ingestion de colostrum, le taux de globulines augmente. De même, Jameson, Alvarez-Tostado Sortor [16] constatent que le sérum de veaux nouveau-nés ne contient pa's de $\gamma$-globulines. Il possède, au contraire, en plus de la séralbumine, une assez forte proportion d' $\alpha$-globulines et de petites quantités de $\beta$-globulines. 18 heures après l'absorption de colostrum, une fraction que l'on identifie à la $\gamma$-globuline, apparaît dans le sang; à la $36^{e}$ heure, sa concentration est identique à celle de l'adulte, mais elle n'acquiert la mobilité de la $\gamma$-globuline adulte que 50 jours après la naissance.

Hansen et Phillips [17] constatent également chez des veaux nouveau-nés nourris au colostrum ou à la pseudo-globuline colostrale, une élévation immédiate des $\gamma$-globulines sériques. Toutefois, si l'animal reçoit le colostrum après la $24^{\mathrm{e}}$ heure, on n'observe pas d'élévation sensible de ces globulines. Au contraire, chez le veau 
nouveau-né, nourri au lait écrémé, les protéines sériques n'atteignent leur taux normal qu'à la $8^{\mathrm{e}}$ semaine; jusqu'à ce moment, on constate une augmentation lente et progressive des $\gamma$-globulines. Forster et coll. [18] confirment sur la truie les résultats de HaNSEN. En particulier, ces auteurs notent dans les 24 premières heures du post-partum l'élévation de la $\gamma$-globuline dans le sérum de cochonnet (de 1 gr. 2 à 20 gr. par litre).

De plus, on a prouvé l'existence d'une maladie hémolytique du muleton (CARoLI et Bessis [19]), du poulain, du chien et du porc, après absorption de colostrum dans les 30 à 36 premières heures de la vie. Dans le cas de l'ictère grave du muleton, des anticorps anti-mulet existent dans le sang et dans le lait de la mère jument immunisée au cours de la grossesse. De même, la transmis. sion par le lait de la maladie hémolytique humaine est possible (Christianns et Goudemand [20]). Les agglutinines complètes anti-A et anti-B existent dans le colostrum pendant une dizaine de jours après la naissance et peuvent être à l'origine d'accidents cliniques en cas d'allaitement maternel (Hirszfeld [21]).

Les anticorps du colostrum jouent donc un rôle très important aussi bien chez l'animal que chez la femme. Le transfert de l'immunité par le colostrum est d'ailleurs un phénomène très curieux. Car il suppose une absorption intestinale des protéines, soit intactes, soit sous forme de gros fragments polypeptidiques. Si les protéines sont hydrolysées par les ferments digestifs, elles donnent naissance à un mélange d'amino-acides et d'oligo-peptides très simples et l'immunité ne peut être transmise. Au contraire, si les protéines ne subissent pas de protéolyse digestive ou si elles sont seulement scindées en gros fragments polypeptidiques (1) et si, d'autre part, elles peuvent traverser sous cette forme la muqueuse intestinale, on peut alors expliquer facilement ce transfert de l'immunité, La question de la traversée de la muqueuse intestinale par des molécules protéiques intactes a d'ailleurs été étudiées expérimentalement par Dent et Schilling [23]. Ces auteurs ont étudié la composition en acides aminés du sang portal de chien partiellement gastrectomisé, prélevé par une canule de London.

Ils ont constaté que le taux d'acides aminés augmentait considérablement après l'ingestion par l'animal de protéines comme la caséine, la sérumalbumine humaine (on observe notamment une augmentation de l'acide glutamique). Au contraire, après l'ingestion d'une protéine homologue (protéine plasmatique de chien) on ne constate pas d'augmentation du taux des acides aminés libres dans

(1) Petermann [22] a montré que les $\gamma$-globulines-anticorps, fragmentées en demimolécules, ou en quarts de molécules, conservaient leur activité immunologique. 
le sang portal. Ceci permet donc de penser que des protéines "homologues" peuvent traverser "intactes" la muqueuse intestinale. СомLINE et coll. [24] ont d'ailleurs démontré le passage direct dans le canal thoracique d'agglutinines (Brucella abortus) provenant de globulines colostrales introduites par une canule dans le duodénum de veaux nouveau-nés anesthésiés. Le passage s'effectue 60 à 120 minutes après l'introduction du colostrum dans le duodénum. Toutefois, ce délai est nettement supérieur chez des animaux âgés de plus de 60 heures.

Mais l'hypothèse du passage direct des globulines immunes à travers la muqueuse intestinale ne peut devenir valable que si l'on admet une inactivité relative des ferments protéolytiques des sucs gastrique et pancréatique vis-à-vis de ces protéines. Dans le cas du colostrum, les globulines anticorps homologues traverseraient la muqueuse intestinale durant les premiers jours ou les premières heures de la vie, peut-être parce que les ferments digestifs sont peu actifs durant cette période. Il existe d'ailleurs dans le colostrum des quantités relativement importantes d'un "inhibiteur de la trypsine ». Cet agent a été purifié et obtenu à l'état cristallisé par LASKowSki et LASkowski [25]. Il diffère de l'«inhibiteur de la trypsine" isolé du suc pancréatique par sa mobilité électropho. rétique, son point isoélectrique, son poids moléculaire et sa composition en acide aminés. Sa présence éviterait l'hydrolyse des globulines immunes dans le tube digestif, tout au moins durant les 24 premières heures de la vie.

A la suite de ces expérimentations modernes, la transmission de l'immunité par le colostrum semble définitivement établie chez l'animal. Chez l'homme elle peut exister également mais associée à une transmission diaplacentaire. L'immunité transmise par cette voie s'expliquerait par la minceur du barrage. Au contraire, chez des animaux comme la truie, la jument, la vache et la chèvre, sept épaisseurs de couches séparent la circulation fotale de la circulation maternelle et gênent le passage des anticorps. Chez la femme, la transmission de l'immunité peut donc emprunter deux voies : diaplacentaire et colostrale. Ceci explique l'utilité du colostrum, associé à des petites transfusions et à des injections de $\gamma$-globulines dans l'élevage des prématurés (MARTIN DU PAN, ScheidegGer et Roulet [26]).

Campbell et coll. [27] ont, d'autre part, émis l'hypothèse que les anticorps du colostrum tireraient leur origine des éléments mobiles du sang, qui viennent s'accumuler en quantité importante dans la glande mammaire au début de la lactation. 


\section{La fraction albumine}

On appelle fraction albumine, la fraction du petit-lait soluble dans le sulfate de magnésium à saturation ou le sulfate d'ammonium à demi-saturation, Elle contient essentiellement trois composants : la $\beta$-lactoglobuline, l' $\alpha$-lactalbumine et la sérumalbumine. Mais en effectuant une étude électrophorétique sur papier à $p \mathbf{H} 8,9$ nous avons pu mettre en évidence, à côté de ces trois constituants principaux, deux composants mineurs $\left(A_{1}\right.$ et $A_{2}$ de la figure 1).

a) La $\beta$-lactoglobuline de Palmer. - Elle représente $55 \%$ des protéines du lactosérum. Ses méthodes de préparation sont nombreuses. Nous citerons notamment :

- la méthode de Palmer [28] qui consiste, après enlèvement de la caséine par acidification et des globulines par précipitation à demi-saturation par le sulfate d'ammonium, à traiter le liquide surnageant dans l'eau et la solution ajustée à $p \mathrm{H} 5,2$ et dialysée à $20^{\circ} \mathrm{C}$. contre de l'eau glacée pendant 10 jours au moins. Au cours de cette dialyse, la $\beta$-lactoglobuline précipite à l'état cristallisé.

- La méthode de Zweig et BLock [29] qui permet d'obtenir la $\beta$-lactoglobuline cristallisée avec d'excellents rendements; les protéines du lactosérum sont précipitées sous forme de complexes ferriques (ferrilactine). Après enlèvement du fer et précipitation fractionnée au sulfate d'ammonium on peut obtenir la $\beta$-lactoglobuline.

Le poids moléeulaire de la $\beta$-lactoglobuline est voisin de 35.000 . L'homogénéité électrophorétique de la $\beta$-lactoglobuline cristallisée est discutable. A certains $p \mathrm{H}$, elle contiendrait deux et quelquefois trois constituants (PoLIs et coll. [30].

En ce qui concerne sa composition en acides aminés, le $\beta$-lactoglobuline est riche en acides aminés dicarboxyliques (19\% d'acide glutamique et $11 \%$ d'acide aspartique) (tableau I) ainsi qu'en leucine et isoleucine. Ces faits rapprochent la $\beta$-lactoglobuline de la sérumalbumine bovine. Mais il existe des différences très marquées entre les deux protéines quant au pourcentage d'autres aminoacides (en particulier taux de méthionine plus élevé et taux de cystine et d'histidine beaucoup moins élevés dans la $\beta$-lactoglobuline.

Par la méthode des DNP-aminoacides de SAnger, Porter [31] a pu établir que la structure primaire de la molécule de $\beta$-lactoglobuline comporte certainement trois chaînes peptidiques terminées toutes par un résidu de leucine possédant son groupement $\alpha$-aminé libre.

b) L'a-lactalbumine. Grâce aux résultats d'ultracentrifugation 
de Svedberg et Pedersen [32], on savait que le petit-lait contenait un composant $\alpha$ que les auteurs ont appelé $\alpha$-lactalbumine. En 1939, SöRENSEN et SöRENSEN [33] ont préparé, à partir de la fraction albumine du petit-lait une protéine cristallisée qu'ils ont appelée "substance cristallisée insoluble ». En 1953, Gordon et Semmet [34] ont observé que les constantes de sédimentation et de diffusion de la substance cristallisée insoluble étaient comparables à celles rapportées par SvedBerg et Pedersen pour le composé $\alpha$; aussi Gordon et SEMmet donnent-ils le nom d' $\alpha$-lactalbumine à la "substance cristallisée insoluble » de SörENSEN.

L' $\alpha$-lactalbumine représente $12 \%$ des protéines totales du petit-lait, Son poids moléculaire est faible : 16.000 environ. Elle est électrophorétiquement homogène. Son taux de tryptophane est particulièrement élevé $(7 \%)$.

\section{Présence dans le lait de sérumalbumine}

Il existe dans le colostrum et le lait parfait, un composant mineur qui représente 3 à $5 \%$ des protéines totales du lactosérum. Ce constituant n'a pu être mis en évidence dans le lait de régression. A partir du lactosérum, il a été séparé sous forme cristallisée par fractionnement aux sels neutres et à l'alcool. Son identité est absolument complète avec la sérumalbumine sanguine (PoLIs et coll. [35]).

(A suivre.)

\section{LA RECHERCHE D'ANTIBIOTIQUES, SEULS OU ASSOCIÉS, DANS LE LAIT (1) \\ par \\ P. DOPTER}

En exposant les conséquences pathologiques, hygiéniques et industrielles $\mathrm{du}$ traitement des infections mammaires par les antibiotiques, JACQUET [1] a signalé une diminution très marquée des mammites dans les régions où la pénicilline était largement (et même abusivement) utilisée. Mais, parmi les affections qui subsistent, on constate une disparition presque complète des formes streptococciques, auparavant dominantes, et une augmentation des formes à staphylocoques et à colibacilles, germes ayant résisté à l'action de la pénicilline. Ce fait a motivé l'emploi de plus'en plus fréquent d'autres antibiotiques, en particulier d'auréomycine et de streptomycine, quelquefois seuls, mais le plus souvent associés à la pénicilline.

(1) Compte rendu Acad. Agric., 1956, 42, no 10, 519. 